\title{
Transport and stirring induced by vortex formation
}

\author{
S. C. SHADDEN ${ }^{1}$, K. KATIJA ${ }^{2}$, M. ROSENFELD ${ }^{3}$, \\ J. E. MARSDEN ${ }^{1}$ AND J. O. DABIRI $I^{2}$ \\ ${ }^{1}$ Control and Dynamical Systems, California Institute of Technology, Pasadena, \\ CA 91125, USA \\ ${ }^{2}$ Graduate Aeronautical Laboratories \& Bioengineering, California Institute of Technology, \\ Pasadena, CA 91125, USA \\ ${ }^{3}$ Department of Fluid Mechanics and Heat Transfer, Tel Aviv University, Ramat Aviv, 69978, Israel
}

(Received 1 December 2006 and in revised form 28 August 2007)

The purpose of this study is to analyse the transport and stirring of fluid that occurs owing to the formation and growth of a laminar vortex ring. Experimental data was collected upstream and downstream of the exit plane of a piston-cylinder apparatus by particle-image velocimetry. This data was used to compute Lagrangian coherent structures to demonstrate how fluid is advected during the transient process of vortex ring formation. Similar computations were performed from computational fluid dynamics (CFD) data, which showed qualitative agreement with the experimental results, although the CFD data provides better resolution in the boundary layer of the cylinder. A parametric study is performed to demonstrate how varying the piston-stroke length-to-diameter ratio affects fluid entrainment during formation. Additionally, we study how regions of fluid are stirred together during vortex formation to help establish a quantitative understanding of the role of vortical flows in mixing. We show that identification of the flow geometry during vortex formation can aid in the determination of efficient stirring. We compare this framework with a traditional stirring metric and show that the framework presented in this paper is better suited for understanding stirring/mixing in transient flow problems. A movie is available with the online version of the paper.

\section{Introduction}

Vortex formation is of interest throughout fluid mechanics. Vortex formation is ubiquitous in turbulence (Pullin \& Saffman 1998); vortex shedding occurs in many engineering applications (e.g. transportation, flow control, etc.) as well as in biological systems (e.g. fish swimming and insect flight; see for example Moore \& Saffman 1973; Weihs 1973; Lugt 1983; Dudley 2002). Vortex formation, growth and eventual shedding into the wake is also of major interest because fluid forces can be computed from the resulting coherent structures (Spedding, Rosen \& Hedenstrom 2003; Dabiri 2005). Instabilities including Richtmyer-Meshkov and the gravity-driven RayleighTaylor flows are dominated by vortex formation at varying length scales (Zabusky 1999).

Because vortex ring formation is prototypical of a broad range of vortex dynamics problems, it is an active area of research with a rich history. Maxworthy (1977) identified the vortex bubble outline of an impulsively ejected slug of fluid, where the 
formation process was inferred from the motion of injected dye. Didden (1979) investigated the vorticity flux using a cylinder apparatus to create vortex rings (which led to a related computational study by Nitsche \& Krasny 1994). Dye visualization techniques were later improved upon by the implementation of particleimage velocimetry (PIV) (Adrian 1991), which was later applied to vortex rings in several studies (e.g. Willert \& Gharib 1991; Gharib, Rambod \& Shariff 1998). In addition to laboratory experiments, numerical computation and development of related theory has made many important contributions to the current understanding of vortex formation (e.g. Pullin 1979; Saffman 1978). Despite these studies, there remain important questions concerning the precise manner in which the vortex forms. The purpose of this study is to analyse the transport mechanisms associated with the formation and growth of a vortex ring. Specifically, we aim to bridge the self-similar roll-up concept adopted in previous theoretical and numerical studies of early vortex formation with the vortex bubble perspective used in experimental investigations of fully formed vortex rings.

We also seek to relate the geometric view of formation to the mixing of the surrounding fluid. Eckart (1948) divided mixing into three separate stages. The first stage of entrainment is characterized by vortical fluid creating large-scale motions. In the following stage of stirring, fluid elements are strained, causing high gradients in concentrations until the large-scale motions are reduced in order to reach that of diffusive processes. Finally, in the third and final stage, the gradients disappear and the molecular mixing process begins. The notion of a quantitative metric for mixing is particularly useful when approaching the control and optimization of mixing. There have been a vast number of mixing measures used for various applications, probably because the quantification of mixing is highly application-specific. Chella \& Ottino (1985), Khakhar \& Ottino (1986) and Khakhar, Rising \& Ottino (1986) provide mixing metrics related to the stirring mechanisms (second stage) that are well documented to govern mixing in laminar flows (see also Mathiew, Mezić \& Petzold 2005).

Because of the challenging nature of flow geometry in unsteady systems, vortex formation in the present study is viewed in terms of coherent structures that develop during the formation process. When the velocity field is steady (and two-dimensional), understanding transport is straightforward. Dabiri \& Gharib (2004) used streamlines to define vortex structures by transforming to a reference frame moving with the vortex ring, but found that when the field was sufficiently unsteady, they could not adequately define the boundary of the ring. These conclusions led to an application of dynamical systems theory, as proposed by Haller (2001) and Shadden, Lekien \& Marsden (2005), to vortex rings, which is described in Shadden, Dabiri \& Marsden (2006). However, the study of Shadden et al. (2006) did not consider the vortex formation process; the present paper addresses this topic. The application of dynamical systems ideas to study the flow geometry of unsteady systems is a growing subject, see e.g. Haller \& Poje (1998), Malhotra, Mezić \& Wiggins (1998), Mezić \& Wiggins (1999), Haller \& Yuan (2000), Haller (2001), Mancho et al. (2003) and Padberg (2005) for examples of related theoretical developments; and Pierrehumbert \& Yang (1993), Poje \& Haller (1999), Jones \& Winkler (2002), Joseph \& Legras (2002), Koh \& Legras (2002), Krasny \& Nitsche (2002), Voth, Haller \& Gollub (2002), Arratia, Voth \& Gollub (2005), Lekien et al. (2005) and Wiggins (2005) for example applications of related methods.

For a vortex generated with a piston-cylinder apparatus, it is intuitive that some portion of the fluid in the cylinder will end up in the vortex, and as the vortex ring grows, ambient fluid will be entrained. We show that Lagrangian coherent structures 


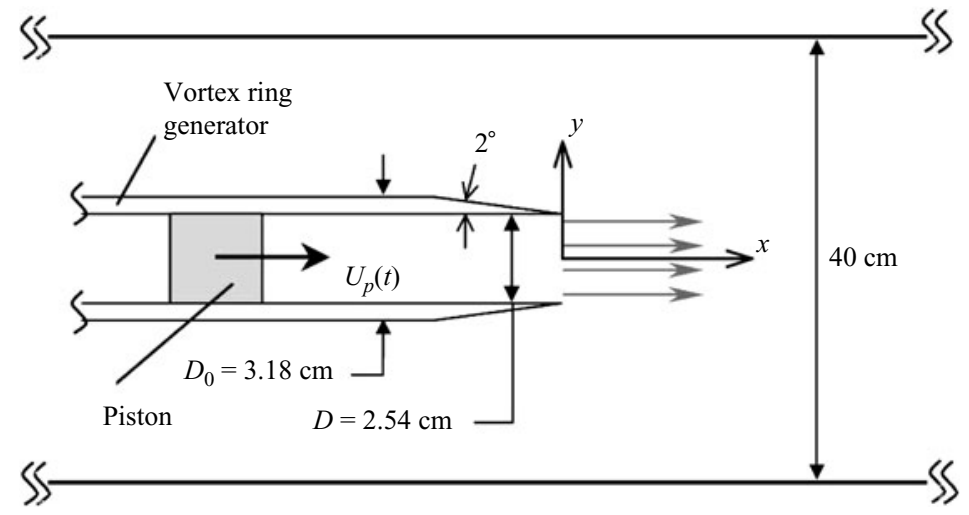

FIgURE 1. Top view of cylinder nozzle exit in tank. Image adapted from Dabiri \& Gharib (2004).

(LCS) determine precisely what fluid inside the cylinder ends up in the vortex bubble and exactly what ambient fluid is entrained. This perspective is used in $\S 4.2 .3$ to determine the time variation of entrainment during formation and how entrainment varies owing to changes in piston-stroke length-to-diameter ratio $(L / D)$. In $\S 5$, we discuss the stirring observed owing to vortex formation. In particular, we study the stretching induced at various interfaces in the flow, relate the observed results to LCS, and compute the average stretching efficiency during formation.

\section{Experimental methods}

Experiments were conducted using a piston-cylinder apparatus similar to the set-up used in Dabiri \& Gharib (2004) (figure 1). A cylinder was placed inside a transparent water tank with dimensions $60 \mathrm{~cm} H \times 40 \mathrm{~cm} W \times 110 \mathrm{~cm} \mathrm{~L}$. A constant pressure head tank $(\Delta p=8.2 \mathrm{kPa})$ drove fluid through a transparent acrylic cylinder with an inner diameter of $2.54 \mathrm{~cm}$ and a outer $2^{\circ}$ taper at the exit. This sharp angle ensured a thin cylinder wall in the domain of interest, which enabled visualization of the flow both inside and outside the cylinder without excess noise from light scatter off the cylinder walls. The apparatus was operated at a nominal flow speed of $5.5 \mathrm{~cm} \mathrm{~s}^{-1}$ and each vortex ring was generated at a piston-stroke length-to-diameter ratio of 2. Based on the cylinder diameter and flow at the exit, the operating Reynolds number was in the laminar regime $(R e=1225)$.

The tank was seeded with $13 \mu \mathrm{m}$ diameter glass spheres. Two pulsed Nd:YAG lasers (New Wave Research) supplied $30 \mathrm{~mJ}$ light pulses at a wavelength of $532 \mathrm{~nm}$. A black-and-white CCD camera (Uniq Vision) was oriented perpendicular to the light sheet and captured the particle motion in the laser plane at $1024 \times 1024$ pixel resolution and 30 frames per second. Based on the location of the laser sheet relative to the camera, a conversion ratio of 86.6 pixels $\mathrm{cm}^{-1}$ was used for subsequent data analysis.

Image pairs were separated by a time interval of $18 \mathrm{~ms}$. Each image was evaluated with an interrogation window size of $32 \times 32$ pixels and a $50 \%$ window overlap. This method produced velocity field data every $1 / 15 \mathrm{~s}$ over a rectangular domain, approximately $[0,20] \times[-10,10]$ in $\mathrm{cm}$, with the exit plane of the cylinder at $x=3.6 \mathrm{~cm}$ and the centreline of the cylinder at $y=0.55 \mathrm{~cm}$. These velocity data were used to compute LCS, as described below. 


\section{Lagrangian method}

To study the geometry of the flow, we employ a Lagrangian criterion based on the distribution of the finite-time Lyapunov exponent (FTLE) field (Shadden et al. 2005) (also sometimes referred to as the direct Lyapunov exponent field in the literature, see Haller 2001). The FTLE measures the maximum stretching about a material point over a chosen time interval. More precisely, let $\phi_{t}^{t+T}: \boldsymbol{x}(t) \mapsto \boldsymbol{x}(t+T)$ denote the mapping of a material point located at $\boldsymbol{x}(t)$ at time $t$ to its position at time $t+T$ under the influence of the fluid motion. The map $\phi_{t}^{t+T}$ is commonly referred to as the flow map. The derivative of the flow map, the deformation gradient,

$$
\frac{\mathrm{d} \phi_{t}^{t+T}(\boldsymbol{x})}{\mathrm{d} \boldsymbol{x}},
$$

is a linear operator that describes how infinitesimal changes of the initial position $\boldsymbol{x}(t)$ influence the final position $\boldsymbol{x}(t+T)$. Defining the finite-time Cauchy-Green deformation tensor as

$$
\Delta(\boldsymbol{x}, t, T)=\frac{\mathrm{d} \phi_{t}^{t+T}(\boldsymbol{x})^{\top}}{\mathrm{d} \boldsymbol{x}} \frac{\mathrm{d} \phi_{t}^{t+T}(\boldsymbol{x})}{\mathrm{d} \boldsymbol{x}},
$$

(where $A^{\top}$ denotes the transpose of $A$ ) allows us to define the FTLE as

$$
\sigma(x, t, T) \triangleq \frac{1}{|T|} \ln \sqrt{\lambda_{\max }(\Delta(\boldsymbol{x}, t, T))}
$$

where $\lambda_{\max }(\Delta(\boldsymbol{x}, t, T))$ denotes the maximum eigenvalue of $\Delta(\boldsymbol{x}, t, T)$. It is easily shown (see Shadden et al. 2005) that a perturbation $\delta(t)$ to $\boldsymbol{x}(t)$ evolves as

$$
\|\boldsymbol{\delta}(t+T)\| \leqslant \exp ^{\sigma(x, t, T)|T|}\|\boldsymbol{\delta}(t)\|,
$$

where equality holds when $\delta(t)$ is aligned with the eigenvector corresponding to $\lambda_{\max }(\Delta(\boldsymbol{x}, t, T))$.

The FTLE field can be used to reveal LCS. Within this framework, LCS are defined as codimension one structures along which the FTLE field is maximized, a notion that is made precise in Shadden et al. (2005). LCS represent almost-invariant boundaries that separate fluid with dynamically different behaviour. For example, it has been shown in Shadden et al. (2006) and Green, Rowley \& Haller (2007) that LCS defined in this manner are an effective and frame-independent way of defining the boundary and structure of vortices in laminar and turbulent flow.

The sign of the integration time, $T$, relates to the type of LCS revealed in the FTLE field. Positive integration times reveal repelling LCS and negative integration times reveal attracting LCS. Additionally, the length of the integration time $|T|$ can be increased or decreased according to the extent of the LCS that must be captured. In many applications, especially when a large integration time is chosen, trajectories leave the velocity domain before the integration time is complete. The domain of the velocity data used in the present study extended far enough for this not to be a concern. However, if trajectories left before the integration time, FTLE for those trajectories, and their immediate neighbours, was computed at the instant before it left the velocity domain. More details on how to choose an appropriate integration time and the computational method can be found in Shadden (2006).

In the results listed herein, time $t$ is non-dimensionalized to $\tau=t \Delta U / \pi D$ so that the results can be interpreted independently of the particular experimental set-up. The parameter $\Delta U$ is the jet velocity relative to the ambient fluid, nominally $5.5 \mathrm{~cm} \mathrm{~s}^{-1}$. The cylinder circumference $\pi D$ serves as the characteristic length scale for the non-dimensionalization. For the current analyses, the absolute time $t=1 \mathrm{~s}$ corresponds to the dimensionless time $\tau=0.69$. 

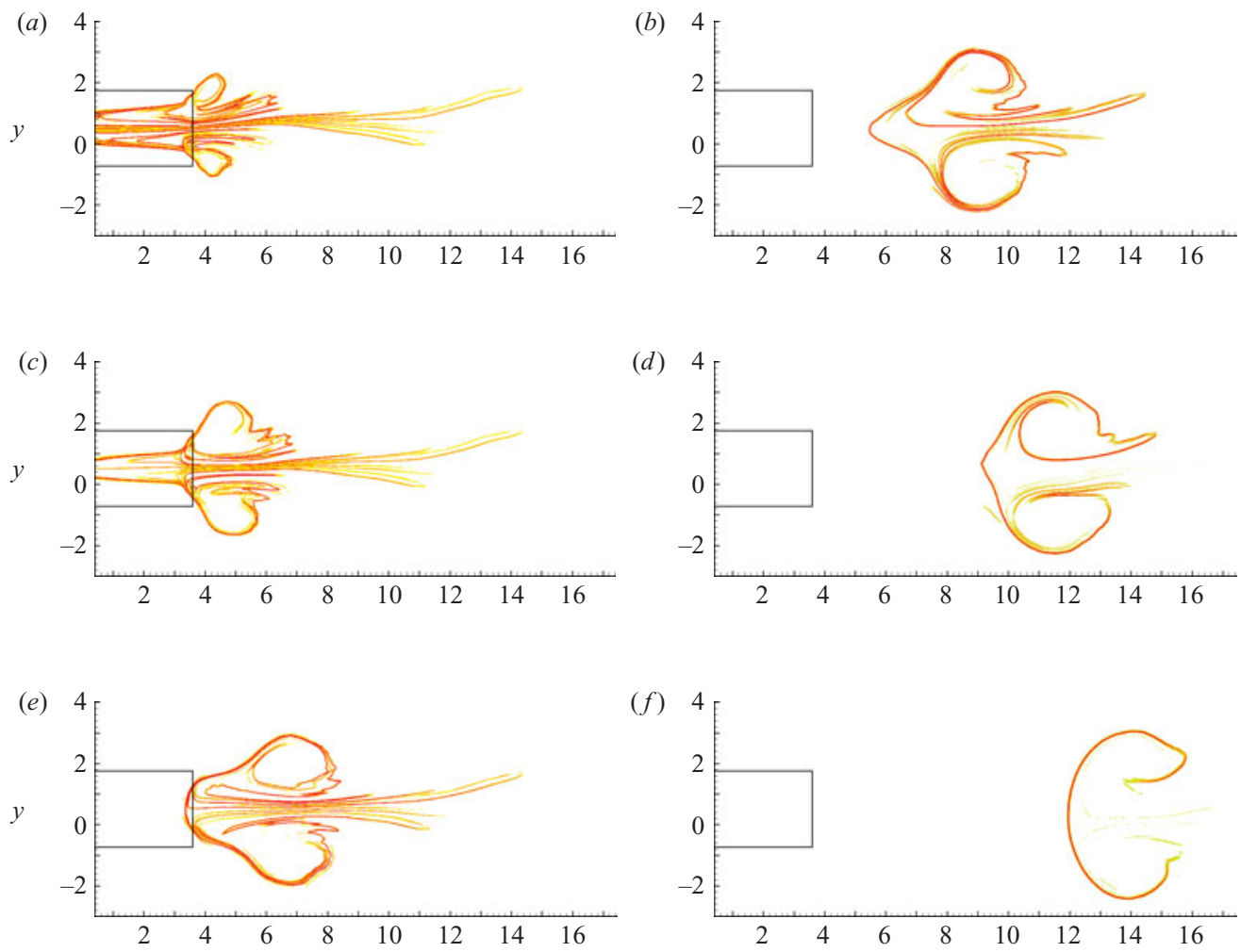

Figure 2. Forward-time FTLE, $\sigma(\boldsymbol{x}, \tau, T)$, fields for vortex formation for various $\tau$. The integration time used was $T=2.78$ for all plots and $x$ - and $y$-axes are plotted in centimetres. The location of the cylinder is given by the solid rectangle. (a) $\tau=0,(b) 1.104,(c) 0.368$, (d) 1.472, (e) 0.736, $(f) 1.840$.

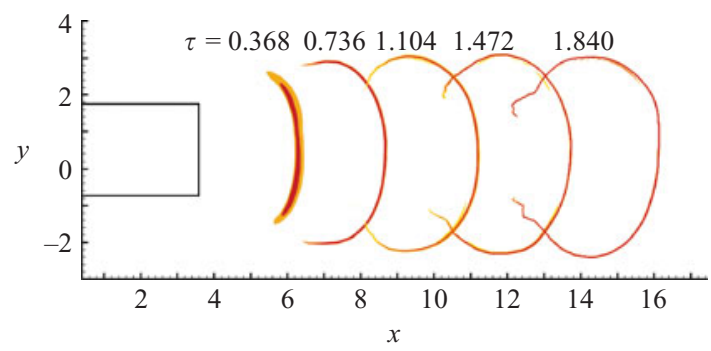

FIGURE 3. Backward-time FTLE plots for vortex formation, with five time instances superimposed on same figure. For each FTLE plot, the integration time was $T=-\tau$.

\section{Flow geometry}

\subsection{Empirical data}

FTLE fields were computed from DPIV data obtained by the methods described in $\S 2$. Figure 2 shows the forward-time FTLE field at a sequence of times, with the cylinder outlined in black. Time $\tau=0$ corresponds to the instant the piston is impulsed. The contour levels in figure 2 are set so that FTLE values below $70 \%$ of the maximum are transparent. The ridge of high FTLE is a repelling LCS. This LCS bounds the fluid that will be entrained into the vortex. 


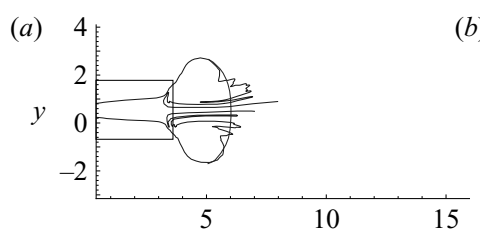

(d)

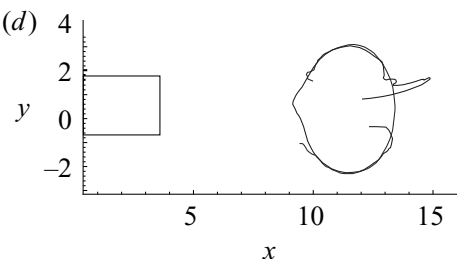

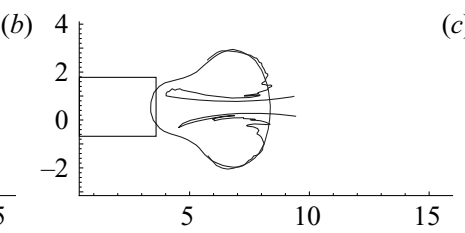

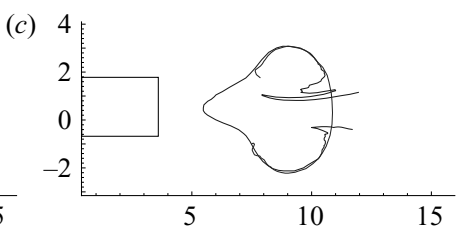

(e)

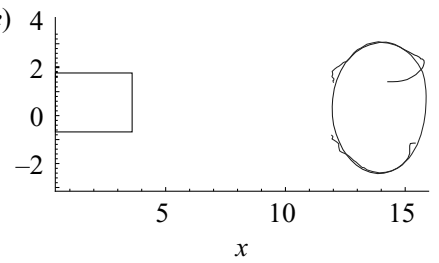

FIGURE 4. Repelling and attracting LCS for vortex formation. $(a) \tau=0.368,(b) 0.736$, (c) $1.104,(d) 1.472,(e) 1.840$. Vortex bubble fully formed by $\tau \approx 1.8$.
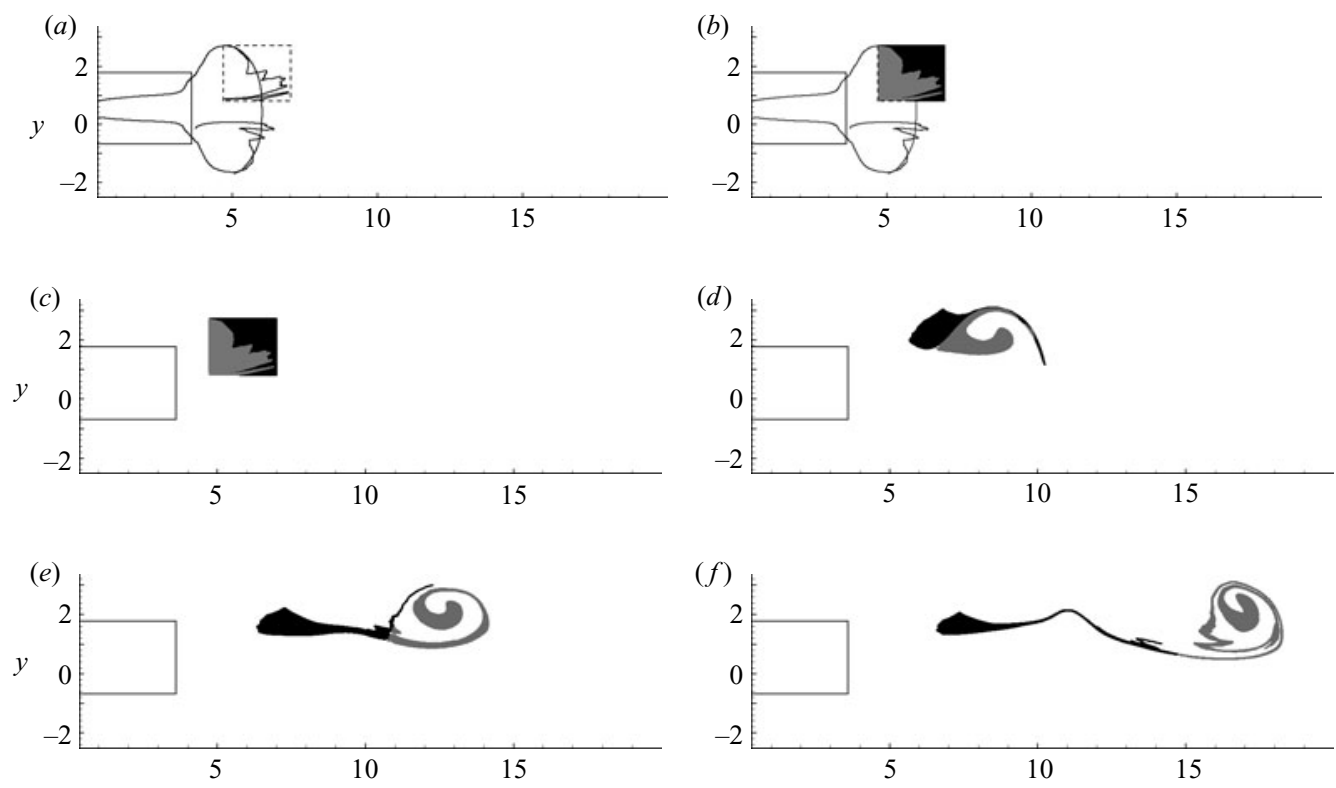

Figure 5. (a) The box (dashed line) marks the boundary of a parcel of tracers that will be released in the flow at $\tau=0.368$. (b) The tracers shaded according to their initial location with respect to the repelling LCS. $(c-f)$ The forward time integration of the parcel.

Similarly, the backward-time FTLE fields were computed from the DPIV data to provide the plots shown in figure 3 (where several snapshots in time are superimposed on the same figure). The attracting LCS revealed from these plots defines the 'front boundary' (downstream side) of the vortex. Since the flow is quiescent for $\tau<0$, the backward-time FTLE is everywhere zero for $\tau \leqslant 0$. Thus, no attracting LCS is present at $\tau=0$. When $\tau \geqslant 0.368$ the attracting and repelling LCS can be superimposed to provide a closed boundary to the forming vortex (figure 4).

To demonstrate that the repelling LCS bounds the fluid that is entrained, we place a parcel of fluid particles in the flow (figure $5 a$ ). The release time, $\tau=0.368$, 
and location are somewhat arbitrary. To aid the visualization, particles are shaded according to their initial location with respect to the repelling LCS (figure $5 b$ ). As these particles are advected, it is clear that all particles inside the repelling LCS are directed into the vortex, and all particles outside are left in the wake. In dynamical systems terminology, there is a persistent hyperbolic trajectory at the rear of the vortex, which is the turning point for particles that recirculate from those that are pushed to the wake. The repelling LCS is the boundary between all particles that are either pushed to the interior of the vortex or pushed to the wake by this turning point. Likewise, there is a persistent hyperbolic trajectory at the front of the vortex and the attracting LCS is the boundary between the particles in front of the vortex and the recirculating particles in the interior of the vortex.

A precise boundary of the vortex during formation is not well-posed. Once the vortex bubble has fully formed, it is natural to define the vortex boundary from the attracting and repelling LCS, as in Shadden et al. (2006). What is relevant during formation is understanding how fluid is entrained into the vortex bubble as it forms. The region bound by the repelling LCS inside the cylinder constitutes the fluid inside the cylinder that ends up in the vortex bubble. It is clear from figure 2( $a)$ that this region constitutes the majority of the fluid initially entrained into the vortex. In $\S 4.2 .3$, we perform a more detailed analysis to quantify entrainment during formation. Following the LCS outside the cylinder, the LCS loops back and forth, forming lobes. Previous studies have shown that the dynamics of such lobes provide the mechanism by which fluid is entrained into fully formed vortex bubbles; see Leonard, Rom-Kedar \& Wiggins (1987); Romkedar, Leonard \& Wiggins (1990); Shariff \& Leonard (1992) and Shadden et al. (2006). It should be noted that these plots show the cross-sectional geometry of a three-dimensional vortex ring. In three-dimensional space, the LCS are two-dimensional surfaces, and these figures can be interpreted as displaying crosssections of those LCS. Because PIV techniques currently limit data collection to planar sections, an empirically based computation of the complete two-dimensional LCS is not possible. However, this is of little consequence for the flow being analysed since the flow is approximately axisymmetric.

Figure 6 compares the Eulerian velocity and vorticity fields with the location of the LCS during the vortex formation. Note that the maximum vorticity is always located inside the boundary given by the LCS, but the LCS give a much clearer understanding of the vortex boundary and how fluid is entrained or detrained.

\subsection{Numerical results}

The DPIV data are not sufficiently resolved to capture flow features in the boundary layer near the cylinder wall. Classical theory predicts that the boundary layer close to the exit plane rolls up and is the primary source of vortex ring circulation. To be geometrically consistent with previous formation studies focusing on roll-up, the repelling LCS should at least partially contain the boundary layer inside the cylinder 'close' to the exit plane. Otherwise, this portion of the boundary layer would not end up in the core of the vortex. However, the LCS deduced from the DPIV data appears to prematurely constrict away from the cylinder wall (see figure $2 a$ ), erroneously suggesting that none of the boundary layer enters the vortex. This is probably due to the inability of the DPIV measurements to resolve boundary-layer effects given the coarseness of the DPIV data, cf. the spatial resolution of the data in figure 6 .

Next we test the hypotheses that the geometric structure of the LCS is robust, and that better measurement resolution allows the LCS to 'hug' the cylinder wall, capturing the boundary layer near the exit, by relying on CFD simulations. 

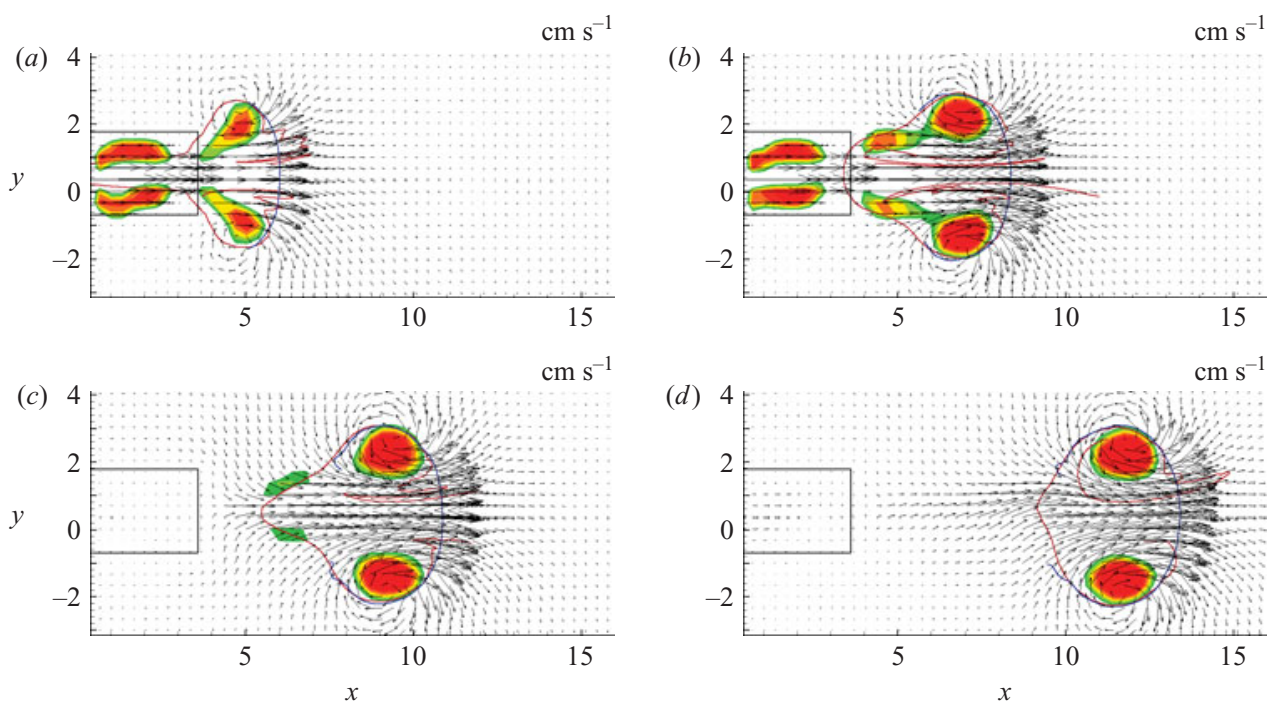

FIGURE 6. DPIV velocity field with LCS and vorticity at a sampling of times, $\tau$. (a) $\tau=0.368$, (b) 0.736, (c) 1.104, (d) 1.472.

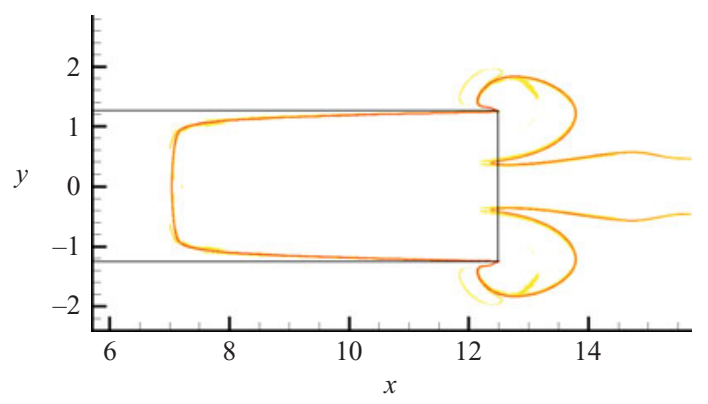

FIGURE 7. Forward-time FTLE field at $\tau=0$ for axisymmetric CFD vortex data with $L / D=2$. The integration time used was $T=3$ and the $x$ - and $y$-axes are plotted in centimetres. The location of the cylinder is represented by the solid rectangle. A complete animation of the FTLE field during vortex formation in available with the online version of the paper.

\subsubsection{CFD method}

An axisymmetric numerical model, similar to the model used in Rosenfeld, Rambod \& Gharib (1998), was constructed with dimensions to match the experimental set-up. The fluid parameters were also set to match the experiment. The downstream boundary was placed at a distance of 15 diameters from the exit plane, while the radial boundary was placed 4 diameters away from the symmetry line. The length of the cylinder was 5 diameters, and the piston was moved with a constant velocity of $5.5 \mathrm{~cm} \mathrm{~s}^{-1}$. No-slip and no-injection velocity was enforced on the walls of the cylinder, while at the outer and downstream boundaries, zero gauge pressure was specified. Zero velocity was specified as the initial condition. Mesh and time step independence tests were used to determine the acceptable mesh and time step, resulting in a mesh of 60000 nodes (clustered near the walls, near the exit of the vortex-ring generator, and in the shear-layer region) and a time step of $2.5 \times 10^{-3} \mathrm{~s}$. The laminar timedependent Navier-Stokes equations were solved using the commercial package Fluent 
(Fluent, Lebanon, NH, USA) employing a second-order spatial and temporal scheme. Although the experiment considered only a piston-stroke length-to-diameter ratio of 2 , numerical simulations were performed for $L / D=0.5,1,2$ and 4 .

\subsubsection{Comparison with experimental results}

Figure 7 shows the forward time FTLE field at $\tau=0$ computed from the CFD data for $L / D=2$, the same $L / D$ as the experimental set-up. The repelling LCS from the empirical data and computational data are qualitatively similar. In both cases, the repelling LCS encloses a slug of fluid inside the cylinder and encloses lobes of fluid outside the cylinder. This resemblance should be expected since LCS are relatively robust to perturbations of the velocity data (Haller 2002). A notable difference is that the repelling LCS computed from the CFD data hugs the wall more closely than the LCS from the empirical data. This confirms the hypothesis that a finer resolution of the velocity data is required near the cylinder wall for the LCS to capture the boundary layer. Also, measurement of the velocity field inside the cylinder is subject to greater measurement error than outside the cylinder owing to unavoidable light scatter from the cylinder wall. This further complicates the ability of the LCS computed from empirical data to capture the boundary layer.

Outside the cylinder, the LCS computed from the empirical data exhibits more spatial oscillation than the LCS from CFD. These oscillations could be due to disturbances in the flow that are inherent to the experimental set-up and are not modelled in the CFD simulations, or due to high-frequency measurement errors. The former is thought to be dominant.

\subsubsection{Entrainment during formation}

The computation of LCS allows insight on how much fluid is entrained during formation. In this section, we compute the amount of fluid that enters the vortex bubble from inside the cylinder, and from outside the cylinder (external fluid), during the formation stage. This information would be difficult to obtain without knowledge of the LCS. In particular, it would be difficult to determine the boundary of the vortex, but also to differentiate cylinder fluid from external fluid over time. For example, empirically visualizing entrainment would be difficult as fluid quickly mixes near the vortex core and diffusion would lead to uncertainty since most common dye tracers possess relatively high Schmidt numbers. Tracking particles by numerical integration would introduce artificial divergence (Tambasco \& Steinman 2002). Using LCS, we can circumvent these issues, as the LCS predict exactly what fluid is entrained.

To compute entrainment during formation, we use CFD data. This allows variation of formation parameters to understand better the effect on entrainment, and allows a more accurate representation of the LCS inside the cylinder, which is important for these computations.

LCS were computed from CFD simulations representing vortex formation for piston-strokes $L / D=0.5,1,2$ and 4. All other parameters discussed in $\S 4.2 .1$ remained constant.

Table 1 gives, for each $L / D$, the amount of fluid bound by the repelling LCS inside the cylinder at $\tau=0$, and, to give a sense of the formation time length, the approximate time at which the vortex boundary attains the prototypical elliptic bubble shape. The values in the second row are computed by taking the (axisymmetric) areas bound by the repelling LCS inside the cylinder and non-dimensionalizing those values by the cross-sectional area of the cylinder. 


\begin{tabular}{cllll}
\multicolumn{1}{c}{$L / D$} & \multicolumn{1}{c}{0.5} & \multicolumn{1}{c}{1} & \multicolumn{1}{c}{2} & 4 \\
Fluid from cylinder & $9.8 \times 10^{-2}$ & $2.8 \times 10^{-1}$ & $6.2 \times 10^{-1}$ & 1.3 \\
Time fully formed & 1.1 & 1.1 & 1.3 & 2.6
\end{tabular}

TABLE 1. Amount of fluid and bubble formation time for different values of $L / D$.
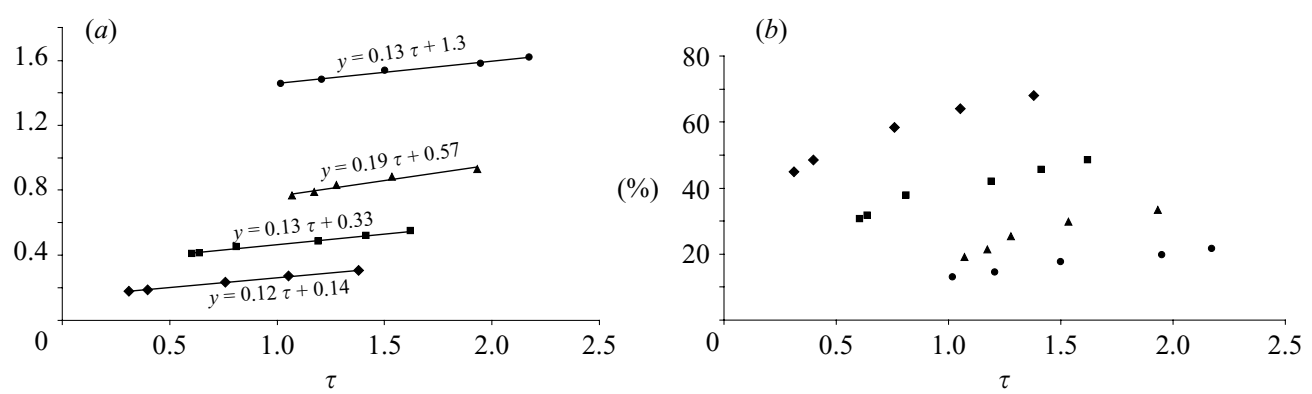

FIGURE 8. (a) Total fluid entrained over time, values represent cross-sectional area normalized by cylinder cross-sectional area. $(b)$ Percentage of entrained fluid from outside the cylinder. $\bullet, L / D=0.5 ; \mathbf{\square}, 1 ; \boldsymbol{\Lambda}, 2 ; \bullet, 4$.

Figure 8(a) plots the total fluid (from inside and outside the cylinder), entrained over time. No detrainment occurs during the time intervals plotted in figure 8 , therefore figure $8(a)$ can be interpreted as the total amount of fluid inside the vortex. The plots do not begin from $\tau=0$ because for $\tau$ small, it is difficult to define precisely the extent of entrained external fluid. Figure 8(a) indicates entrainment is approximately linear. Since the fluid from the cylinder is always included, this entrainment is from external fluid. Furthermore, if we compare the $y$-intercept for the linear best fit to the amount of fluid from the cylinder (see table 1), we see that the entrainment is relatively linear between $\tau=0$ and the first data points. For $L / D$ from 0.5 to 2 , the rate of entrainment (i.e. slope of the linear fit) appears to increase, and then slightly decrease from $L / D=2$ to $L / D=4$.

Figure $8(b)$ displays the relative contribution of entrained external fluid to the total vortex volume. This plot demonstrates that relative to the size of the vortex, smaller $L / D$ leads to more entrainment of external fluid. This conclusion is consistent with measurements of Müller \& Didden (1980) and Dabiri \& Gharib (2004).

\section{Stirring and mixing}

\subsection{Interface stretching}

Next we discuss the amount of stretching induced at various interfaces in the flow during formation in order to shed light on the use of pulsatile or vortical flows for mixing. It is well-known that the mixing of two fluids is greatly enhanced by increasing the interface between the fluids (Aref 2002; Ottino 1989). The increase in the interface between two fluids (stirring) allows for a larger area for diffusion to occur (mixing). For this analysis, we use the empirical flow data. Similar conclusions are found by analysis of the computational flow data.

The first interface, I1, is located across the exit plane of the cylinder, representative of a fluid inside a chamber being injected into another fluid (figure $9 a$ ). The other two 


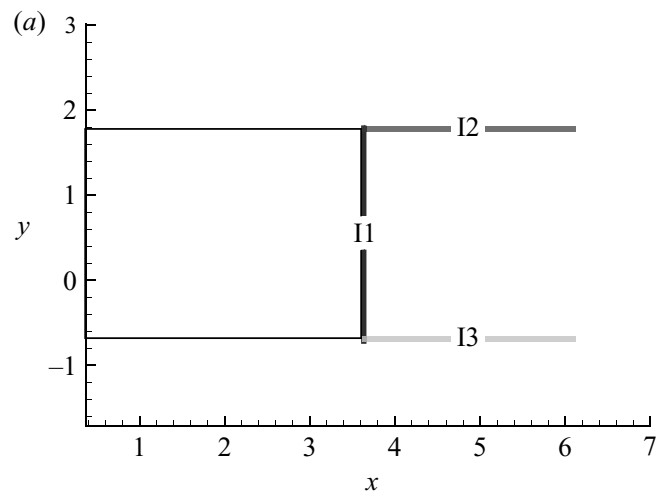

(b)
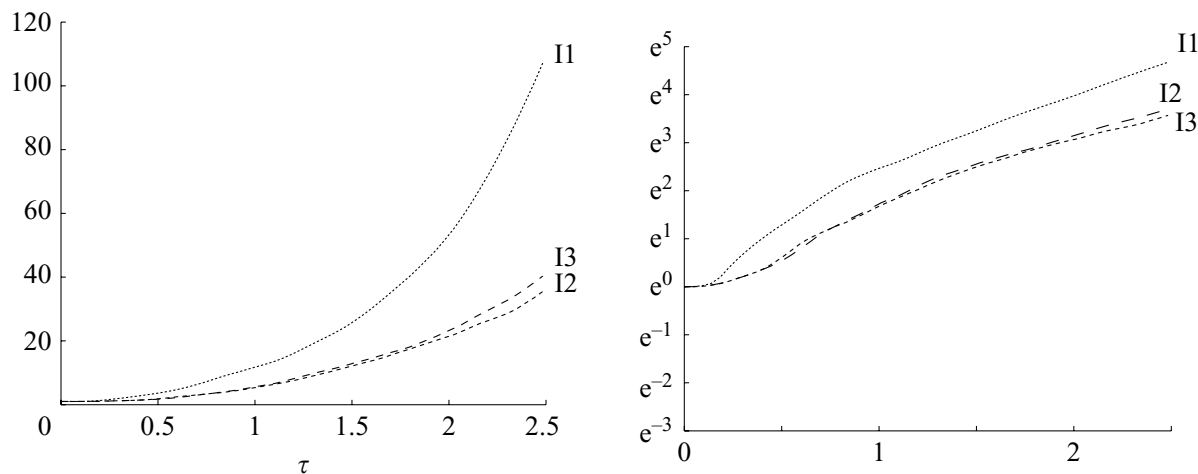

FiguRE 9. (a) Location of interfaces used to study mixing. The cylinder outline is shown by the thin black line. (b) Stretching factor of interface versus time, $\tau=0$ corresponds to initialization of vortex formation.

interfaces, I2 and I3, are located, respectively, along the top and bottom edge of the cylinder, downstream from the exit plane. Their location coincides with the location of the shear layer (in a time-averaged sense). For comparison, interfaces I1-I3 are initially the same length, equal to the cylinder diameter. Figure $9(b)$ shows the length of each interface over time, plotted on a linear and natural log scale. The lengths plotted in figure $9(b)$ are normalized by the initial length of the interface, therefore the graph represents the stretch factor over time.

The stretching of I1, I2 and I3 appears exponential. Interface I1 has the highest exponential growth during formation. The growth rates of interfaces I2 and I3 are similar to each other, which should be expected since the vortex ring is approximately axisymmetric.

The discrepancy in the stretching between I1 and I 2 or I3 can be better understood by considering the repelling LCS. Figure $10(a)$ superimposes the forward-time FTLE field with the locations of the material interfaces at $\tau=0$. As discussed previously, there is hyperbolic trajectory at the rear of the vortex that acts as a turning point between fluid that recirculates and fluid left in the wake; the repelling LCS defines the boundary between this fluid. Thus a fluid parcel placed over the repelling LCS will be (exponentially) stretched apart. If an interface intersects a repelling LCS, then we can expect this to result in high stretching. If the interface intersects this structure multiple times, then this influence is multiplied. 


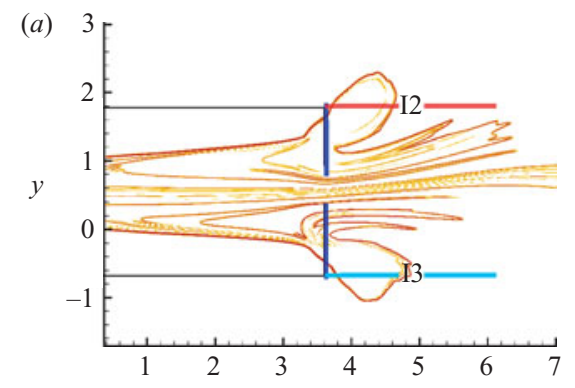

(b)
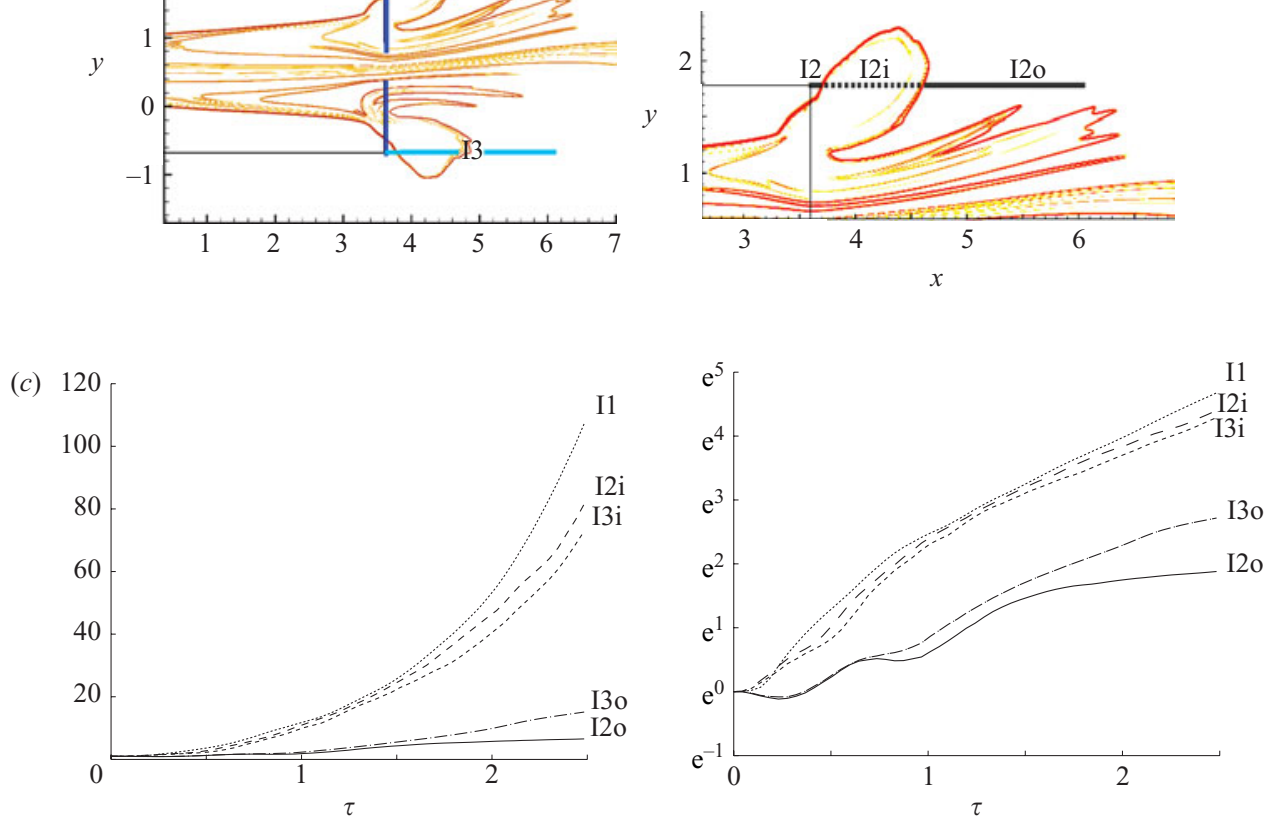

FIGURE 10. (a) Location of interfaces used to study mixing with respect to initial location of repelling LCS. (b) Portion of I2 initially inside vortex, I2i, and portion of I2 initially outside vortex, I2o. (c) Stretching factors for I2i, I2o, I3i and I3o, stretching factor for I1 shown for reference.
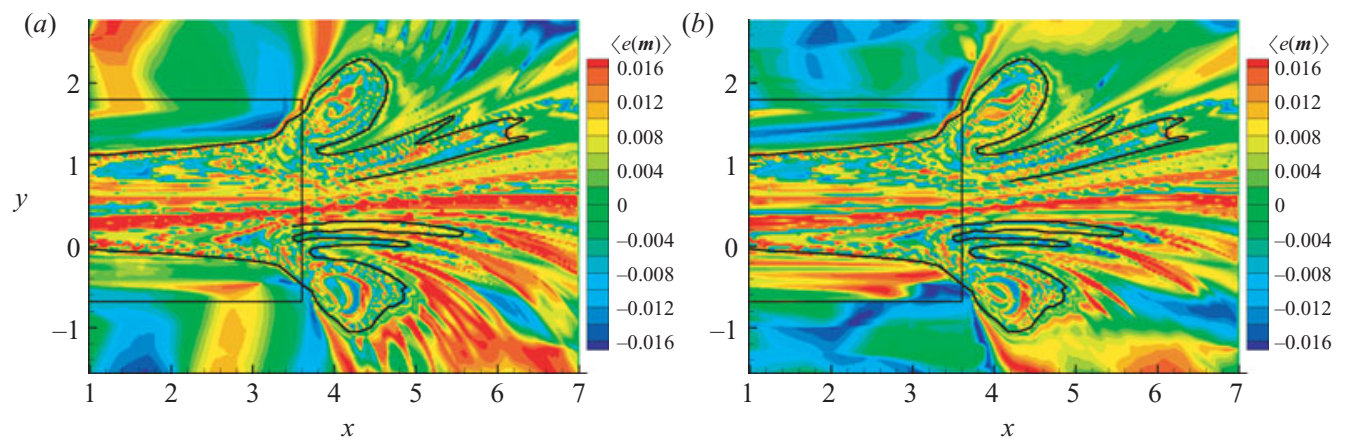

FIGURE 11. Average mixing efficiency field in $(a)$ the $x$-direction and $(b)$ the $y$-direction. For reference, the LCS at $\tau=0$ is superimposed on the efficiency fields (solid black curve).

It is clear that interface I1 intersects the LCS multiple times and I2 or I3 intersect the LCS less than I1. Therefore we can expect the stretching of I1 to be higher than the other two interfaces, which is consistent with the data of figure $9(b)$.

Note that most of the stretching of I 2 and I 3 is due to the segments intersecting the region bound by the repelling LCS. The segment of I 2 intersecting the area enclosed by LCS at $\tau=0$ is denoted by the dotted line (I2i) and the segment located outside 
this region is denoted by the solid line (I2o) in figure $10(b)$. Interface I3 can be similarly partitioned to give corresponding segments I3i and I3o. The growth rates of these individual segments are shown in figure $10(c)$, where again each segment length is normalized by its length at $\tau=0$ to be interpreted as the stretching factor over time. The stretching factor of I1 over time is also plotted in figure $10(c)$ for comparison. The stretching of $\mathrm{I} 2 \mathrm{i}$ and $\mathrm{I} 3 \mathrm{i}$ is substantially greater than for I2o or I3o. In fact, the stretching of $\mathrm{I} 2 \mathrm{i}$ and $\mathrm{I} 3 \mathrm{i}$ is very close to the stretching of $\mathrm{I} 1$ during the formation stage, i.e. $\tau<1.5$. As we continue along the LCS, further intersections of the LCS with I1 are present, which lead to the increasing rate of stretching of I1 over I $2 \mathrm{i}$ or I3i during the remainder of the time interval considered.

As the integration time used to compute the FTLE increases, more of the LCS is revealed. In addition, the LCS cannot intersect itself. As we follow the repelling LCS away from the rear hyperbolic trajectory, the LCS progressively loops backward and forward. When it loops backward, it is confined by the previous segment, which leads to an accumulation of lobes in the interior of the vortex. For example, this leads to lobes within lobes, which shows how fluid that is initially entrained is later detrained. Although I2 or I3 appear to intersect the repelling LCS only twice, if the integration time used to compute the FTLE field were increased, more of the LCS would be revealed and more intersections would probably be apparent inside the area intersected by $\mathrm{I} 2 \mathrm{i}$. Since the integration time required to reveal this portion of the LCS would be greater than 2.54 (the integration time used to compute the field shown), the influence of these intersections is most noticeable after the time interval presently considered. However, it is only in this region bound by the LCS that continued stretching induced by the vortex circulation will persist.

We see that the location of an interface with respect to an LCS plays a pivotal role in predicting stirring. Once the LCS are computed, they tell us directly the best locations in the flow to introduce different fluids to be mixed. This offers a perspective that does not require tracking interfaces, a task that becomes increasingly difficult, in both experiments and simulations, as the fluid becomes well stirred. In addition to revealing where stirring occurs, this perspective also allows us to understand better the mechanisms of stirring.

\subsection{Mixing measures}

There are a number of mixing measures that have been used in the literature to characterize how well a fluid mixes. No single measure is best suited for all applications because the measures are highly dependent upon factors such as importance of diffusion, chemical reaction, miscibility, domain of interest, etc. The present study is focused on kinematic phenomena and thus neglects diffusion or reacting fluids. A parameter that was developed to gauge such kinematic mixing is the average stretching efficiency (see Khakhar et al. 1986; Ottino 1990). Here, we apply this criterion to evaluate mixing for the empirical vortex formation.

Before defining the average stretching efficiency we first define the stretch factor

$$
\rho(\boldsymbol{x}, t, T)=\lim _{\|\boldsymbol{\delta}(t)\| \rightarrow 0} \frac{\|\boldsymbol{\delta}(t+T)\|}{\|\boldsymbol{\delta}(t)\|},
$$

that is, the factor by which the magnitude of an infinitesimal perturbation $\delta(t)$ to an arbitrary point $\boldsymbol{x}(t)$ grows in time. Note that if the initial perturbation $\delta(t)$ is aligned with the eigenvector of the maximum eigenvalue of $\Delta(\boldsymbol{x}, t, T)$, then $\rho(\boldsymbol{x}, t, T)=\sqrt{\lambda_{\max }(\Delta(\boldsymbol{x}, t, T))}$, see $\S 3$. Also, if $\boldsymbol{\delta}(t)$ is thus aligned, the FTLE field 
can be rewritten as

$$
\begin{aligned}
\sigma(\boldsymbol{x}, t, T) & =\frac{1}{|T|} \ln \rho(\boldsymbol{x}, t, T) \\
& =\frac{1}{|T|} \int_{t}^{t+T} \frac{\rho^{\prime}(\boldsymbol{x}, t, s)}{\rho(\boldsymbol{x}, t, s)} \mathrm{d} s,
\end{aligned}
$$

where $\rho^{\prime}$ denotes differentiation with respect to the third slot (e.g. the variable $s$ ).

The average stretching efficiency can be defined in terms of the specific rate of stretch, $\rho^{\prime} / \rho$, as

$$
\langle e(\boldsymbol{x}, \boldsymbol{m}, t, T)\rangle=\int_{t}^{t+T} \frac{\rho^{\prime} / \rho}{\sqrt{\mathbf{S}: \mathbf{S}}} \mathrm{d} s,
$$

where $\boldsymbol{S}$ is the symmetric part of the linearized velocity field,

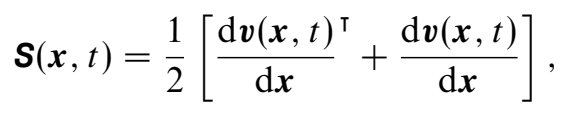

and $\boldsymbol{S}: \boldsymbol{S}$ represents the tensor inner product (contraction). The quantity $\sqrt{\boldsymbol{S}: \boldsymbol{S}}$ is the rate of viscous dissipation in a Newtonian fluid. Therefore it allows comparison between the action of fluid stretching and the action of viscosity, i.e. it tells how much energy is lost in the process of the stretching. Using

$$
\dot{\boldsymbol{\delta}}(t)=\frac{\mathrm{d} \boldsymbol{v}(\boldsymbol{x}, t)}{\mathrm{d} \boldsymbol{x}} \boldsymbol{\delta}(t),
$$

it readily follows that

$$
\frac{\rho^{\prime}}{\rho}=\langle\boldsymbol{m}, \boldsymbol{S m}\rangle,
$$

where $\boldsymbol{m}=\boldsymbol{\delta}(t) /\|\boldsymbol{\delta}(t)\|$, which makes explicit the dependence of the stretching efficiency on $\boldsymbol{m}$. That is, the stretching efficiency is dependent on the orientation of the perturbation $\delta(t)$ used to compute it.

The average stretching efficiencies in the $x$ - (axial) and $y$ - (radial) direction are plotted in figure 11, where we have chosen $\boldsymbol{m}$ and $\boldsymbol{n}$ to denote unit vectors in the $x$ - and $y$-directions, respectively. The efficiency fields shown are for $\tau=0$ with an integration/averaging time of $T=2.622$ (after 2.622 time units, particles begin to leave the velocity data domain). These plots also contain the repelling LCS at $\tau=0$ for comparison. The average efficiency fields have some similarity to the FTLE fields, which might be expected since the FTLE is related to the average efficiency, as derived above. It is clear, however, that the average efficiency fields do not provide sharp boundaries as the FTLE fields, thus making interpretation of the efficiency fields difficult. Additionally, the efficiency fields show regions of high efficiency outside the repelling LCS, which is misleading since we know from $\S 5.1$ that stirring is poor in these regions. This is probably due to the denominator $\sqrt{\boldsymbol{s : S}}$ being small in these regions where the flow has little motion or is approximately quiescent.

It is instructive to consider how the average efficiency field changes with the integration (averaging) time, $T$. Figure 12 shows the spatial maximum of the average efficiency field $\langle e(\boldsymbol{m})\rangle$ versus the integration time. The spatial maximum of the average efficiency field in the $y$-direction versus $T$ is nearly identical to the plot in figure 12 . Notice that the stretching efficiency is highly dependent on the integration time for $T<1$. This is one indication that the average stretching efficiency is not a good indicator of mixing for transient flows. Secondly, we notice that the maximum 


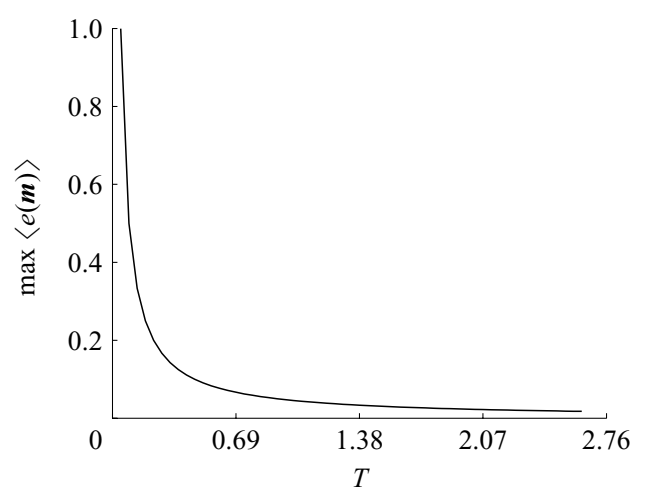

FIGURE 12. Change in the maximum of the average efficiency field versus the integration time.

efficiency asymptotes to a very small, if not zero, value with increasing integration time, implying that the field becomes homogeneous, and hence void of useful information. This is probably due to the vortex having only a local, in time, influence on the domain shown, and eventually the stretching induced by the vortex formation no longer causes stirring in this domain, either because the vortex ring has detrained all particles initially in this domain, or simply the vortex ring is too far downstream.

It can be shown that irrespective of the integration time used to compute the average stretching efficiency field, the metric erroneously predicts high kinematic mixing where we know kinematic mixing is low. Furthermore, the mechanism for kinematic mixing is not clearly revealed from the average stretching efficiency field. Although it is not possible to apply all existing kinematic mixing metrics, we expect other measures of kinematic mixing to have similar performance. Such methods typically assume periodicity or some sort of ergodicity, which is often not present in many applications, including the application studied here.

\section{Conclusions}

The purpose of this paper was to provide a clear understanding of the flow structure during vortex-ring formation, and demonstrate how stirring is induced by vortex formation. Intuitively, we expect that during the formation of a piston-cylinder generated vortex ring, much of the fluid in the cylinder will end up in the vortex, and as the vortex propagates, external fluid will be entrained. We demonstrated that LCS can be used to determine precisely what fluid is entrained to form the vortex, and how that entrainment occurs over time.

Demonstrating the process of vortex formation from this perspective aids in the qualitative understanding of the formation process, but can also be exploited to derive quantitative information that would otherwise be difficult to obtain. In $\S 4.2 .3$, be we computed LCS from CFD simulations to determine the overall entrainment volume during formation and the rate of entrainment of fluid outside the cylinder. The pistonstroke length-to-diameter ratio was varied to study its influence on entrainment. Lower $L / D$ was shown to increase the relative contribution of entrained external fluid.

In $\S 5.1$, the stretching at various interfaces is analysed for the empirical data. It is shown that LCS can be used to guide the introduction of fluid(s) into the flow in order to achieve maximum stirring. We also apply a common stirring metric, the average stretching efficiency, to study the empirical flow data. The effectiveness of this metric 
was limited owing to the transient nature of the problem. Other common measures of kinematic mixing are often based on instantaneous deformations or asymptotic distributions in time, making such measures poorly suited for unsteady flows, or those that have a transient effect on the domain of interest.

The authors would like to acknowledge funding from NSF OCE-0623475 (to J. O. D.) and from NSF grant IIS-0413078 (to J. E. M.).

\section{REFERENCES}

Adrian, R. J. 1991 Particle-imaging techniques for experimental fluid-mechanics. Annu. Rev. Fluid Mech. 23, 261-304.

Aref, H. 2002 The development of chaotic advection. Phys. Fluids 14 (4), 1350-1325.

Arratia, P. E., Voth, G. A. \& Gollub, J. P. 2005 Stretching and mixing of non-Newtonian fluids in time-periodic flows. Phys. Fluids 17 (5), 053102.

Chella, R. \& Ottino, J. M. 1985 Stretching in some classes of fluid motions and asymptotic mixing efficiencies as a measure of flow classification. Arch. Rat. Mech. Anal. 90, 15-42.

DABIRI, J. O. 2005 On the estimation of swimming and flying forces from wake measurements. J. Exp Biol. 208, 3519-3532.

Dabiri, J. O. \& Gharib, M. 2004 Fluid entrainment by isolated vortex rings. J. Fluid Mech. 511, $311-331$.

DidDEN, N. 1979 On the formation of vortex rings: rolling-up and production of circulation. Z. Angew. Math. Phys. 30, 101-116.

Dudley, R. 2002 The Biomechanics of Insect Flight: Form, Function, Evolution. Princeton University Press.

ECKART, C. 1948 An analysis of the stirring and mixing processes in incompressible fluids. J. Mar. Res. 7, 265-275.

Gharib, M., Rambod, E. \& Shariff, K. 1998 A universal time scale for vortex ring formation. J. Fluid Mech. 360, 121-140.

Green, M. A., Rowley, C. W. \& Haller, G. 2007 Detection of Lagrangian coherent structures in three-dimensional turbulence. J. Fluid Mech. 572, 111-120.

Haller, G. 2001 Distinguished material surfaces and coherent structures in three-dimensional fluid flows. Physica D 149, 248-277.

HALler, G. 2002 Lagrangian coherent structures from approximate velocity data. Phys. Fluids 14 (6), 1851-1861.

Haller, G. \& Poje, A. C. 1998 Finite time transport in aperiodic flows. Physica D 119, 352-380.

Haller, G. \& Yuan, G. 2000 Lagrangian coherent structures and mixing in two-dimensional turbulence. Physica D 147, (3-4), 352-370.

Jones, C. K. R. T. \& WinkleR, S. 2002 Invariant manifolds and Lagrangian dynamics in the ocean and atmosphere. In Handbook of Dynamical Systems (ed. B. Fiedler), vol. 2, pp. 55-92. Elsevier.

Joseph, B. \& Legras, B. 2002 Relation between kinematic boundaries, stirring, and barriers for the Antarctic polar vortex. J. Atmos. Sci. 59, 1198-1212.

KhakhaR, D. V. \& Ottino, J. M. 1986 Fluid mixing (stretching) by time periodic sequences of weak flows. Phys. Fluids 29 (11), 3503-3505.

Khakhar, D. V., Rising, H. \& Ottino, J. M. 1986 Analysis of chaotic mixing in two model systems. J. Fluid Mech. 172, 419-451.

KoH, T. Y. \& Legras, B. 2002 Hyperbolic lines and the stratospheric polar vortex. Chaos 12, 382-394.

Krasny, R. \& Nitsche, M. 2002 The onset of chaos in vortex sheet flow. J. Fluid Mech. 454, 47-69.

Lekien, F., Coulliette, C., Mariano, A. J., Ryan, E. H., Shay, L. K., Haller, G. \& Marsden, J. 2005 Pollution release tied to invariant manifolds: a case study for the coast of Florida. Physica D 210, 1-20.

Leonard, A., Rom-Kedar, V. \& Wiggins, S. 1987 Fluid mixing and dynamical systems. Proc. Intl Conf. on the Physics of Chaos and Systems Far from Equilibium, Nuclear Physics B 2, 179-190.

Lugt, H. J. 1983 Vortex Flow in Nature and Technology. John Wiley. 
Malhotra, N., Mezić, I. \& Wiggins, S. 1998 Patchiness: a new diagnostic for Lagrangian trajectory analysis in time-dependent fluid flows. Intl J. Bifurcation Chaos 8, 1053-1093.

Mancho, A. M., Small, D., Wiggins, S. \& IDE, K. 2003 Computation of stable and unstable manifolds of hyperbolic trajectories in two-dimensional, aperiodically time-dependent vector fields. Physica D 182, 188-222.

Mathiew, G., Mezić, I. \& Petzold, L. 2005 A multiscale measure for mixing. Physica D 211, $23-46$.

Maxworthy, T. 1977 Some experimental studies of vortex rings. J. Fluid Mech. 81, 465-495.

Mezić, I. \& Wiggins, S. 1999 A method for visualization of invariant sets of dynamical systems based on the ergodic partition. Chaos 9, 213-218.

Moore, D. W. \& Saffman, P. G. 1973 Axial flow in laminar trailing vortices. Proc. R. Soc. Lond. A 333, 491-508.

Müller, E. A. \& DidDEN, N. 1980 Zur erzeugung der zirkulation bei der bildung eines ringwirbels an einer dusenmundung. Stroj. Casop. 31, 363-372.

Nitsche, M. \& Krasny, R. 1994 A numerical study of vortex ring formation at the edge of a circular tube. J. Fluid Mech. 276, 139-161.

Ottino, J. M. 1989 The Kinematics of Mixing: Stretching, Chaos, and Transport. Cambridge University Press.

Ottino, J. M. 1990 Mixing, chaotic advection, and turbulence. Annu. Rev. Fluid Mech. 22, 207-253.

PADBerg, K. 2005 Numerical analysis of chaotic transport in dynamical systems. PhD thesis, University of Paderborn.

Pierrehumbert, R. T. \& Yang, H. 1993 Global chaotic mixing on isentropic surfaces. J. Atmos. Sci. 50, 2462-2480.

Poje, A. C. \& Haller, G. 1999 Geometry of cross-stream mixing in a double-gyre ocean model. J. Phys. Oceanogr. 29, 1649-1665.

Pullin, D. I. 1979 Vortex ring formation at tube and orifice openings. Phys. Fluids 22, 401-403.

Pullin, D. I. \& Saffman, P. G. 1998 Vortex dynamics in turbulence. Annu. Rev. Fluid Mech. 30, $31-51$.

Romkedar, V., Leonard, A. \& Wiggins, S. 1990 An analytical study of transport, mixing and chaos in an unsteady vortical flow. J. Fluid Mech. 214, 347-394.

Rosenfeld, M., Rambod, E. \& Gharib, M. 1998 Circulation and formation number of laminar vortex rings. J. Fluid Mech. 376, 297-318.

SaffMAn, P. G. 1978 The number of waves on unstable vortex rings. J. Fluid Mech. 84, 721-733.

SHAdDEN, S. C. 2006 A dynamical systems approach to unsteady systems. PhD thesis, California Institute of Technology.

Shadden, S. C., Lekien, F. \& Marsden, J. E. 2005 Definition and properties of Lagrangian coherent structures from finite-time Lyapunov exponents in two-dimensional aperiodic flows. Physica D 212, 271-304.

Shadden, S. C., Dabiri, J. O. \& Marsden, J. E. 2006 Lagrangian analysis of fluid transport in empirical vortex rings. Phys. Fluids 18 (4), 0471051-11.

Shariff, K. \& LeONARD, A. 1992 Vortex rings. Annu. Rev. Fluid Mech. 24, U235-U279.

Spedding, G. R., Rosen, M. \& Hedenstrom, A. 2003 A family of vortex wakes generated by a thrush nightingale in free flight in a wind tunnel over its entire natural range of flight speeds. J. Exp. Biol. 206, 2313-2344.

Tambasco, M. \& Steinman, D. A. 2002 On assessing the quality of particle tracking through computational fluid dynamic models. J. Biomech. Engng 124, 166-175.

Voth, G. A., Haller, G. \& Gollub, J. P. 2002 Experimental measurements of stretching fields in fluid mixing. Phys. Rev. Lett. 88, 254501.

WeIHS, D. 1973 Hydromechanics of fish schooling. Nature 241, 290-291.

WigGins, S. 2005 The dynamical systems approach to Lagrangian transport in oceanic flows. Аnnu. Rev. Fluid Mech. 37, 295-328.

Willert, C. E. \& Gharib, M. 1991 Digital particle image velocimetry. Exps Fluids 10 (4), 181-193.

ZABUSKY, N. J. 1999 Vortex paradigm for accelerated inhomogeneous flows: visiometrics for the Rayleigh-Taylor and Richtmyer-Meshkov environments. Annu. Rev. Fluid Mech. 31, 495-536. 